Available online at www.eccomasproceedia.org

Eccomas Proceedia COMPDYN (2021) 1458-1475

ECCOMAS

Proceedia
COMPDYN 2021

$8^{\text {th }}$ ECCOMAS Thematic Conference on Computational Methods in Structural Dynamics and Earthquake Engineering M. Papadrakakis, M. Fragiadakis (eds.) Streamed from Athens, Greece, 28 - 30 June 2021

\title{
MULTI-RESOLUTION FINITE WAVELET DOMAIN METHOD FOR FAST TRANSIENT DYNAMIC ANALYSIS IN HOMOGENEOUS AND HETEROGENEOUS RODS AND BEAMS
}

\author{
Dimitris K. Dimitriou ${ }^{1}$, Christos V. Nastos ${ }^{2}$ and Dimitris A. Saravanos ${ }^{3}$ \\ Dept. of Mechanical Engineering \& Aeronautics, University of Patras \\ GR-26500, Rion-Patras, Greece \\ 1e-mail: d.dimitriou@upnet.gr \\ 2 e-mail: xnastos@upatras.gr \\ 3 e-mail: saravanos@mech.upatras.gr
}

\begin{abstract}
A Multi-resolution wavelet-based numerical method is developed for the fast prediction of transient response in elastic homogeneous and heterogeneous rods and beams. The method takes advantage of the remarkable mathematical properties of Daubechies wavelet and scaling functions as basis functions for the spatial approximation of state variables. The Multiresolution capability of the Daubechies wavelet family, provides the hierarchical computational framework that incorporates both scaling and wavelet functions. An uncoupled solution system between each resolution is formulated, using an explicit time integration scheme. The first level of analysis provides the coarse solution, while finer approximations are sequentially calculated and superimposed on the coarse solution, until the desired level of accuracy is achieved, without discarding the previous results obtained at coarser resolutions. Additional$l y$, due to the orthogonality and compact support of Daubechies wavelet family, the decoupled mass matrices of each resolution are diagonal, or block diagonal and the stiffness matrices are banded. The proposed method uses a uniform grid which remains practically unchanged when increasing the order of interpolation (p-method), owing to its meshless character. Numerical results for the simulation of high-frequency wave propagation in isotropic and orthotropic rods and beams are presented and compared against confirmed models, demonstrating substantial reduction in computational effort. Furthermore, additional benefits of the proposed method are shown, such as the localization capabilities of the fine solutions, which exhibit high sensitivity in detecting discontinuities resulted from material inhomogeneity.
\end{abstract}

Keywords: Daubechies wavelets, Multi-resolution analysis, Wavelet-based elements, Composite structures, Guided waves. 


\section{INTRODUCTION}

The transient dynamic response of isotropic and composite materials and structures is attracting intensive research over the last decade, as it is central to classical problems and applications. For instance, the design of passive and active Structural Health Monitoring (SHM) systems based on linear guided waves is an emerging technology, which requires rapid and robust simulation tools for ultrasonic wave propagation in healthy and damaged structures. However, the modeling of wave propagation in light-weight structures is computationally demanding, because of the high frequencies and wavenumbers involved in SHM applications. In addition, the anisotropy and inhomogeneity of composite light-weight structures, adds further complexity and makes the analysis of such problems even more challenging. Traditional methods, such as the Finite Element (FE) and Finite Difference Method, require very fine spatial and temporal discretization, mandated by the small wavelengths and the high frequencies. Remeshing techniques are implemented for the increase of the results accuracy, but using the h- or p-refinement method requires the introduction of intermediate nodes which render the simulation of Lamb or guided waves practically intractable in terms of required computation time. Computational efficiency is further deteriorated in damage detection applications, where most of the widely used detection methods have been developed independently, without sharing common features and synergies with numerical methods.

A powerful and multifunctional mathematical tool, which is mainly used in signal processing, pattern recognition and data compression, is wavelet analysis. During the past three decades, various researchers have studied the use of wavelets to solve partial differential equations (PDEs) [1]. Also, numerous works have investigated the application of several wavelets in computational mechanics and structural analysis. Patton and Marks [2] created a wavelet-based rod element for static and free vibration problems. Ma et al. [3] constructed a beam element based on Daubechies scaling functions, that exhibited great results in static analyses. Diaz et al. [4] have also developed a Daubechies wavelet-based Euler-Bernoulli beam element and a Mindlin-Reisner plate element for static problems. Except from Daubechies wavelets, Deslauries-Dubuc interpolating wavelets, known as interpolets, have been employed for the construction of beam elements for static [5] and wave propagation analysis [6]. Ko, Kurdila and Pilant [7] have developed a class of finite elements based on orthonormal compactly supported wavelets, and used Daubechies wavelets to solve a second order Neumann problem. In a different direction, wavelets have been used in dynamic problems for the reduction of PDEs to ODEs [8],[9] and for the development of wavelet-based time integration schemes [10]. Furthermore, B-spline wavelets have been studied for the construction of rod, beam, and plate elements for static and dynamic problems [11],[12],[13],[14],[15]. Moreover, Hermitian interpolation wavelets have been exploited for the simulation of wave propagation in isotropic structures [16]. Nastos et al. have implemented the Daubechies scaling functions and introduced the Finite Wavelet Domain (FWD) method for the simulation of wave propagation problems in rods, beams, membranes and plates [17],[18],[19]. Due to the orthogonality of Daubechies scaling functions, diagonal or block diagonal consistent mass matrices are created, and the equivalent wavelet domain algebraic system is rapidly solved using explicit time integration, evincing high accuracy and convergence rates. The FWD method incorporated first order shear and higher order layerwise theories either for beam or for plate structures, to achieve rapid and efficient simulation of symmetric and antisymmetric guided waves in thin and thick laminated composites.

All the forenamed wavelet-based methods are confined to the utilization of the scaling functions of each wavelet family; hence they can be termed as single-resolution (SR) approaches. However, the most distinct property of many wavelet families is the Multi- 
resolution (MR) property. This property has mainly been investigated by numerous scientists in the fields of image and signal processing, feature extraction, data compression and denoising, et cetera [20],[21]. During the last decade, many researchers implemented the MR approximation in the field of computational mechanics. Quraishi and Sandeep [22] have created customized second generation wavelets that lead to a scale-decoupled system for the hierarchical static analysis of beams and plates, because of the stiffness decoupling. Jang et al. [23] used hat interpolation wavelets to solve Neumann and Dirichlet boundary value problems. Liu et al. [24] have developed a wavelet MR interpolation Galerkin method for the targeted enrichment of the solution of plane elastic problems. Also, several research works in the field of fracture mechanics using wavelet MR approaches have also been reported [25],[26],[27]. Additionally, Shen, Li and Ou [28] have studied the wave dispersion characteristics of 2D elastic waves using multiscale B-spline wavelets on interval (BSWI) method.

The present work takes advantage of the MR property of Daubechies wavelet family, to develop a hierarchical computational method as an expansion of the FWD method. The proposed method is termed as Multi-resolution Finite Wavelet Domain method (MR-FWD) and employs both Daubechies wavelet and scaling functions as basis functions for the generalized displacement field approximation. Due to the orthogonality and the MR property of Daubechies wavelet family, the solution system is uncoupled for each resolution in the wavelet space. In the present paper, the concept of the MR-FWD method is introduced for 1D cases, and its performance and functionalities are evaluated towards the simulation of longitudinal and guided waves in rods and beams.

In the following sections, the concept of the MR analysis and the mathematical properties of Daubechies scaling functions (SF) and wavelet functions (WF) are introduced. Then, the development of the MR-FWD approximation for rods and beams is described and the hierarchical solution scheme is formulated using explicit time integration. The final physical solution consists of the coarse resolution component and the superposition of sequential finer resolution components that incrementally improve the accuracy of the analysis. Finally, numerical results for transient dynamic analysis in homogeneous and inhomogeneous rod and beam structures are presented and evaluated.

\section{MATHEMATICAL PROPERTIES OF DAUBECHIES WAVELET FAMILY}

Daubechies scaling functions have been employed as basis functions for the FWD and other methods, owing to their remarkable mathematical properties [21],[29]. The Daubechies wavelet family consists of compactly supported orthogonal scaling functions $\varphi(x)$ and wavelet functions $\psi(x)$. Both SF (also called father wavelet) and WF (also called mother wavelet) are needed to form the Multi-resolution approximation. Also, both SF, WF and their derivatives are algorithmically constructed and do not have analytical expressions [30]. Many researchers have studied the computation of wavelet integrals, also termed as connection coefficients, which is a challenging task because of the highly oscillatory and noisy nature of many SF and WF [7],[31],[32]. The basic mathematical properties of Daubechies SF and WF are summarized below.

Compact Support. Both SF and WF are bounded within an interval, which spans over a compact support domain of grid points $[0,2 \mathrm{~L}-1]$, where $\mathrm{L}$ is the order of the SF/WF. For the sake of conciseness, Daubechies WF/SF of order L are termed as DBL. This can be visualized in Figure 1. The values of SF and WF beyond their support are zero by definition. So, the influence of each SF/WF is limited within their support domain, that depends on the order L. Also, SF/WF of order L can be defined in the wavelet domain, either by translating the parent 
$\mathrm{SF} / \mathrm{WF}$ at other integer points $j$, thus creating new SF/WF $\phi(x-j), \psi(x-j)$, or by shrinking/dilating the parent functions to form new ones $\phi\left(2^{j} x\right), \psi\left(2^{j} x\right)$.

Orthogonality. The integer translates of the SF/WF are orthogonal to each other, hence, they form an orthogonal basis in the functional space,

$$
\begin{gathered}
\int_{-\infty}^{\infty} \varphi(x-i) \varphi(x-j) d x=\delta_{i j} \\
\int_{-\infty}^{\infty} \psi(x-i) \psi(x-j) d x=\delta_{i j} \\
\int_{-\infty}^{\infty} \varphi(x-i) \psi(x-j)=0
\end{gathered}
$$

Vanishing moments. The number of vanishing moments signifies the maximum degree of polynomial that can be exactly approximated by SF/WF. Daubechies SF/WF of order L have $\mathrm{L}$ vanishing moments, which are the highest amongst first generation wavelets. It should be noted, that a SF/WF with $\mathrm{L}$ vanishing moments can exactly represent polynomials up to L-1 order.

Two scale relation/ Dilation property. Provided by the dilation equations,

$$
\begin{gathered}
\varphi\left(2^{j} x\right)=\sqrt{2} \cdot \sum_{k=0}^{2 L-1} h_{k} \cdot \varphi\left(2^{j+1} x-k\right) \\
\psi\left(2^{j} x\right)=\sqrt{2} \sum_{k=0}^{2 L-1}(-1)^{k} h_{2 L-k-1} \cdot \varphi\left(2^{j+1} x-k\right)
\end{gathered}
$$

where $h_{k}$ is the set of $2 \mathrm{~L}$ filter coefficients, and $\mathrm{j}$ is the resolution or scale. The dilation property constitutes the basis for the MR analysis since it mathematically connects the scaling and the wavelet function. Also, SF and WF can be calculated at dyadic points from the dilation equations.

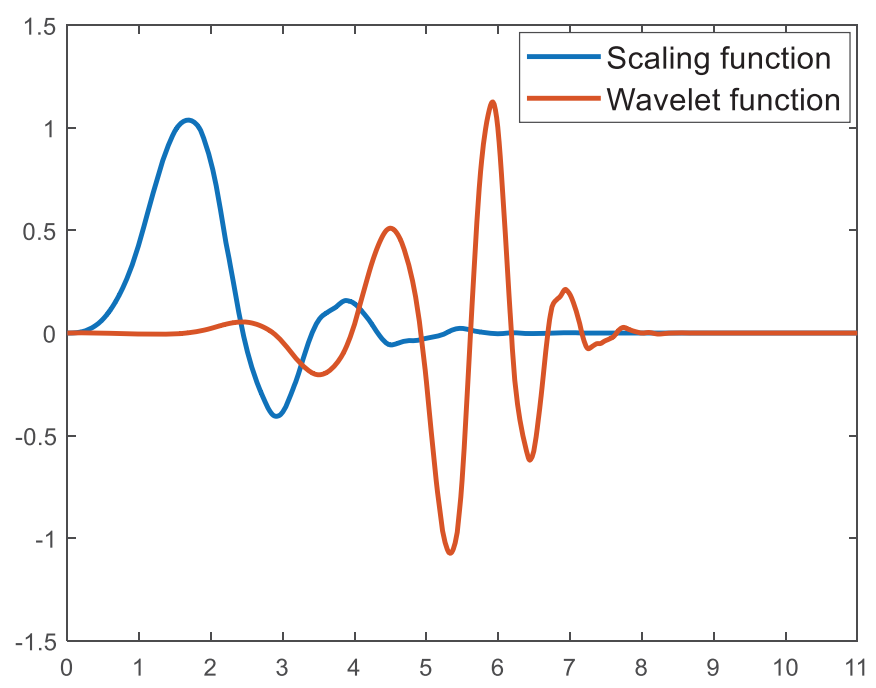

Figure 1: Example of Daubechies scaling $\varphi(\mathrm{x})$ and wavelet $\psi(\mathrm{x})$ functions of order $\mathrm{L}=6$ (DB6). 


\section{THE 1D MULTI-RESOLUTION FINITE WAVELET DOMAIN METHOD}

In the present section, the basic concepts of the MR-FWD method for 1D problems and the construction of MR wavelet-based elements for rods and beams are presented. Besides that, the MR hierarchical procedure using explicit time integration scheme and the achievement of effective resolution decoupling are described.

\subsection{Generalized approximation of field variables}

For a generalized 1D problem, Figure 2 depicts a rod or beam structure that is subdivided into 2 segments, using a uniform grid of 3 grid points. Additional 2L-2 nodes (where L is the order of the SF/WF) are introduced at the left side of the physical domain, and so the wavelet domain is formed. The case of DB3 discretization is shown in Figure 2, so the additional nodes are 4 . The approximation of the generalized displacements $u(x, t)$ in the segment between 2 grid points, also called a wavelet-based element, for $R$ resolutions can be expressed as:

$$
u(x, t)=\sum_{n=-(2 L-2)}^{0} \hat{u}_{C n}^{0}(t) \varphi(\xi-n)+\sum_{n=-(2 L-2)}^{0}\left\{\sum_{S=0}^{R} \hat{u}_{F n}^{S}(t) \psi\left(2^{s} \xi-n\right)\right\}
$$

where $\mathrm{L}$ is the order of Daubechies SF/WF, $\hat{u}_{C n}^{0}$ are the coarse wavelet coefficients of generalized displacements at resolution 0 , and $\hat{u}_{F n}^{S}$ are the fine wavelet coefficients of generalized displacements at resolution S. Also, apart from the global coordinate system, a normalized local coordinate system is associated with each element, as shown in Figure 2. The local dimensionless coordinate variable $\xi$ is given in terms of the global coordinate variable $x$ as:

$$
\xi=\frac{x-x_{i}}{x_{i+1}-x_{i}}=\frac{x-x_{i}}{l_{e}}
$$

so, in Equation (6) the spatial variables $x$ and $\xi$ are limited to the ranges $0 \leq x \leq l_{e}$ and $0 \leq \xi \leq 1$, respectively. Also, $l_{e}$ is the elemental length.

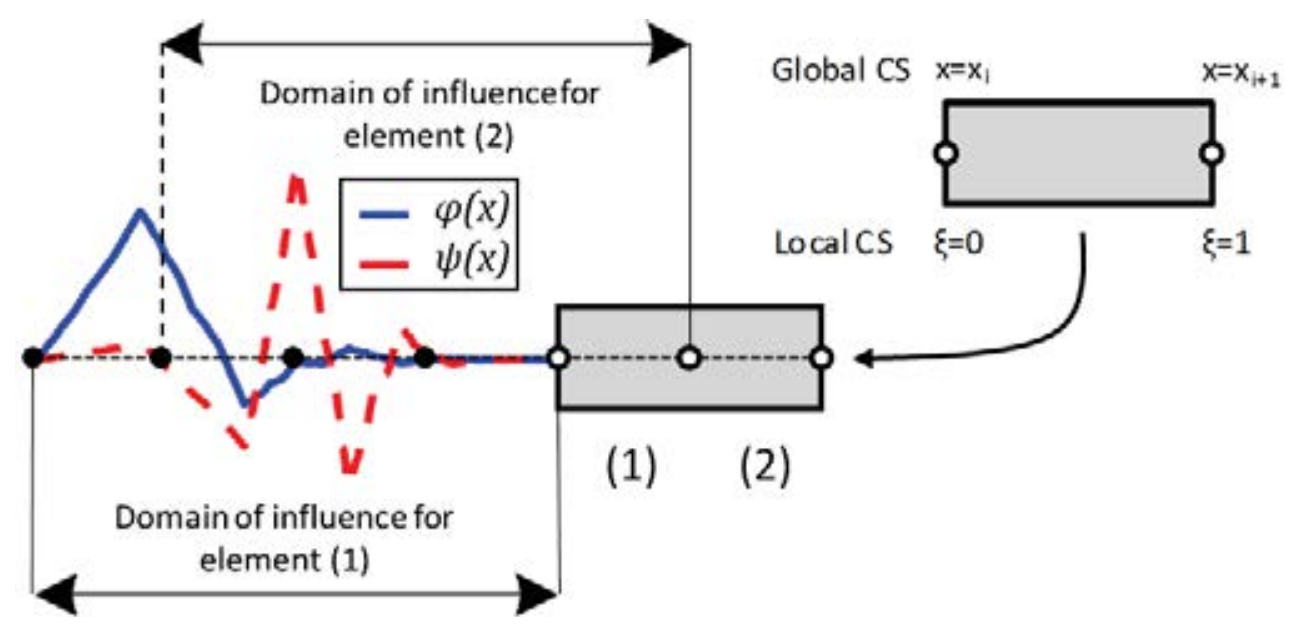

Figure 2: Discretization of the wavelet domain for 2 DB3 MR wavelet-based elements.

\subsection{MR-FWD Rod-Beam element}

The aforementioned MR approximation is implemented for the construction of Timoshenko beam elements. It should be noted, that the rod element is basically included in the beam element, since the rod kinematics are comprehended in the first shear deformation theory 
(FSDT). Specifically, the proposed wavelet-based shear beam element encompasses transverse displacement and rotational degrees of freedom, except from axial displacement. According to the FSDT, the axial and transverse displacement, $u$ and $w$ respectively are expressed by the following equations:

$$
\begin{gathered}
u(x, z, t)=u^{0}(x, t)+\beta_{x}(x, t) \cdot z \\
w(x, z, t)=w^{0}(x, t)
\end{gathered}
$$

where $u^{0}$ and $w^{0}$ are the axial and transverse displacement at the beam midplane, respectively, and $\beta_{x}$ is the rotation of the neutral line. The axial and shear strain, $\varepsilon_{x}$ and $\varepsilon_{x z}$, respectively, are calculated as:

$$
\begin{gathered}
\varepsilon_{x}(x, z, t)=\varepsilon_{x}^{0}(x, t)+k_{x}(x, t) \cdot z \\
\varepsilon_{x z}(x, z, t)=\varepsilon_{x z}^{0}(x, t)
\end{gathered}
$$

in which $\varepsilon_{x}^{0}=u{ }_{,}^{0}, k_{x}=\beta_{x},{ }_{x}$ and $\varepsilon_{x z}^{0}=\beta_{x}+w_{, x}^{0}$. The generalized Hooke's law takes the form:

$$
\left\{\begin{array}{l}
N_{x} \\
N_{x z} \\
M_{x}
\end{array}\right\}=\left[\begin{array}{ccc}
A_{11} & B_{11} & 0 \\
B_{11} & A_{55} & 0 \\
0 & 0 & D_{11}
\end{array}\right]\left\{\begin{array}{l}
\varepsilon_{x}^{0} \\
\varepsilon_{x z}^{0} \\
k_{x}
\end{array}\right\} \Leftrightarrow \sigma_{g e n}=[A B D] \varepsilon_{g e n}
$$

where $N_{x}, N_{x z}$ and $M_{x}$ are the axial force, shear force and bending moment, and $A_{11}, A_{55}, B_{11}$ and $D_{11}$ are the extensional, shear, coupling and flexural stiffness terms, respectively. As shown in Eq. (12) the generalized Hooke's law can take compact form, introducing the generalized stress $\sigma_{g e n}=\left[N_{x}, N_{x z}, M_{x}\right]^{T}$, the generalized strain $\varepsilon_{g e n}=\left[\varepsilon_{x}^{0}, \varepsilon_{x z}^{0}, k_{x}\right]^{T}$ and the wellknown $[A B D]$ matrix of the beam. The Principle of Virtual Work provides the variational form of the equation of motion for the FSDT beam:

$$
-\int_{0}^{l} \delta \varepsilon_{\text {gen }}^{T} \cdot \sigma_{\text {gen }} d x+\int_{0}^{l} \delta u_{\text {gen }}^{T} \cdot \rho_{\text {total }} \cdot \ddot{u}_{\text {gen }} d x+\int_{S} \delta u_{\text {gen }}^{-T} \cdot \bar{\tau} d S=0
$$

where $u_{\text {gen }}=\left[u^{0}, w^{0}, \beta_{x}\right]^{T}$ are the generalized displacements, $\rho_{\text {total }}=\operatorname{diag}\left[\rho^{A}, \rho^{A}, \rho^{D}\right]$ is the diagonal total density matrix of the beam, in which $\rho^{A}$ is the linear density and $\rho^{D}$ is the rotational inertia, and overbar variables are tractions and displacement on the boundary $S$. The approximation of generalized displacements of a MR-FWD shear beam element for R resolutions is:

$$
\left\{\begin{array}{l}
u^{0} \\
w^{0} \\
\beta_{x}
\end{array}\right\}=\sum_{n=-(2 L-2)}^{0}\left\{\begin{array}{l}
\hat{u}_{C n}^{0} \\
\hat{w}_{C n}^{0} \\
\hat{\beta}_{x C n}
\end{array}\right\} \cdot \varphi(\xi-n)+\sum_{S=0}^{R} \sum_{n=-(2 L-2)}^{0}\left\{\begin{array}{l}
\hat{u}_{F n}^{0, S} \\
\hat{w}_{F n}^{0, S} \\
\hat{\beta}_{x F n}^{0, S}
\end{array}\right\} \cdot \psi\left(2^{S} \xi-n\right)
$$

and the generalized strains for resolution $\mathrm{R}$, are expressed as:

$$
\left\{\begin{array}{l}
\varepsilon_{x}^{0} \\
\varepsilon_{x z}^{0} \\
k_{x}
\end{array}\right\}=\sum_{n=-(2 L-2)}^{0}\left[\Phi,,_{x}\right] \cdot\left\{\begin{array}{l}
\hat{u}_{C n}^{0} \\
\hat{w}_{C n}^{0} \\
\hat{\beta}_{x C n}
\end{array}\right\}+\sum_{S=0}^{R} \sum_{n=-(2 L-2)}^{0}\left[\Psi,,_{x}\right] \cdot\left\{\begin{array}{l}
\hat{u}_{F n}^{0} \\
\hat{w}_{F n}^{0} \\
\hat{\beta}_{x F n}
\end{array}\right\}
$$


where matrix $\Phi_{, x}$ is the coarse strain shape function matrix:

$$
\left[\Phi,,_{x}\right]=\frac{1}{l_{e}}\left[\begin{array}{ccc}
\varphi, \xi(\xi-n) & 0 & 0 \\
0 & \varphi_{,_{\xi}}(\xi-n) & l_{e} \cdot \varphi(\xi-n) \\
0 & 0 & \varphi, \xi(\xi-n)
\end{array}\right]
$$

and matrix $\Psi_{, x}$ is the fine strain shape function matrix for resolution R:

$$
\left[\Psi,,_{x}\right]=\frac{1}{l_{e}}\left[\begin{array}{ccc}
\psi,_{\xi}\left(2^{S} \xi-n\right) & 0 & 0 \\
0 & \psi,,_{\xi}\left(2^{S} \xi-n\right) & l_{e} \cdot \psi\left(2^{S} \xi-n\right) \\
0 & 0 & \psi,_{\xi}\left(2^{S} \xi-n\right)
\end{array}\right]
$$

The implementation of Eqs. (14), (15), (16) and (17) to Eq. (13) yields the MR stiffness and mass matrices of the shear beam element. For simplicity, the hierarchical MR process for resolution 1 will be presented, considering that the generalized displacement field is termed as $u$ in the physical space.

Resolution $1\left(C^{0}+F^{0}\right)$. Following the hierarchical MR scheme, the fine approximation at resolution $0\left(F^{0}\right)$ needs to be calculated and added to the coarse solution at resolution $0\left(C^{0}\right)$ in order to obtain $C^{l}$ solution. The MR discretized solution system is:

$$
\left[\begin{array}{cc}
M_{C C} & 0 \\
0 & M_{F F}
\end{array}\right]\left\{\begin{array}{l}
\ddot{\hat{u}}_{C}(t) \\
\ddot{\hat{u}}_{F}(t)
\end{array}\right\}+\left[\begin{array}{cc}
K_{C C} & K_{C F} \\
K_{F C} & K_{F F}
\end{array}\right]\left\{\begin{array}{l}
\hat{u}_{C}(t) \\
\hat{u}_{F}(t)
\end{array}\right\}=\left[\begin{array}{l}
F_{C}(t) \\
F_{F}(t)
\end{array}\right]
$$

where $\hat{u}_{F}$ are the generalized fine wavelet coefficients and $\hat{u}_{C}$ are the generalized coarse wavelet coefficients of the coupled equations of motion for resolution 1 . The coarse solution at resolution $0\left(C^{0}\right)$ is obtained assuming that $\hat{u}_{F}=0$. In that way, this single-resolution coarse solution, termed as $\hat{u}_{C C}$, is basically the same solution that the FWD method provides, employing only the Daubechies SF $\varphi(x)$. It is important to point out that $\hat{u}_{C}$ is not equal to $\hat{u}_{C C}$, because of the coupling between the coarse and fine equations of motion. That coupling emanates from the coupling stiffness matrices $\left[K_{C F}\right]$ and $\left[K_{F C}\right]$ that are nonzero in opposition to $\left[M_{C F}\right]$ and $\left[M_{F C}\right]$ that are equal to zero because of the orthogonality between $\varphi(x)$ and $\psi(x)$. Also, $\left[K_{F F}\right]$ and $\left[M_{F F}\right]$ are the fine resolution stiffness and mass matrices, respectively, and $F_{F}$ is the fine resolution load vector. More specifically, all the aforementioned MR stiffness and mass matrices are calculated as:

$$
\left[K_{C C}^{e}\right]=\left[\begin{array}{ccc}
\frac{A_{11}}{l_{e}} \Gamma_{k l}^{11} & \frac{B_{11}}{l_{e}} \Gamma_{k l}^{11} & 0 \\
& \frac{A_{55}}{l_{e}} \Gamma_{k l}^{11} & A_{55} \Gamma_{k l}^{01} \\
(S) & & A_{55} l_{e} \Gamma_{k l}^{00}+\frac{D_{11}}{l_{e}} \Gamma_{k l}^{11}
\end{array}\right]
$$




$$
\begin{aligned}
& {\left[K_{C F}^{e}\right]=\left[\begin{array}{ccc}
\frac{A_{11}}{l_{e}} \Gamma 1_{k l}^{11} & \frac{B_{11}}{l_{e}} \Gamma 1_{k l}^{11} & 0 \\
& \frac{A_{55}}{l_{e}} \Gamma 1_{k l}^{11} & A_{55} \Gamma 1_{k l}^{01} \\
(S) & & A_{55} l_{e} \Gamma 1_{k l}^{00}+\frac{D_{11}}{l_{e}} \Gamma 1_{k l}^{11}
\end{array}\right]} \\
& {\left[K_{F C}^{e}\right]=\left[K_{C F}^{e}\right]^{T}} \\
& {\left[K_{F F}^{e}\right]=\left[\begin{array}{ccc}
\frac{A_{11}}{l_{e}} \Gamma 2_{k l}^{11} & \frac{B_{11}}{l_{e}} \Gamma 2_{k l}^{11} & 0 \\
\frac{A_{55}}{l_{e}} \Gamma 2_{k l}^{11} & A_{55} \Gamma 2_{k l}^{01} \\
(S) & & A_{55} l_{e} \Gamma 2_{k l}^{00}+\frac{D_{11}}{l_{e}} \Gamma 2_{k l}^{11}
\end{array}\right]} \\
& {\left[M_{C C}^{e}\right]=\rho_{\text {total }} \cdot l_{e} \cdot \Gamma_{k l}^{00}} \\
& {\left[M_{F F}^{e}\right]=\rho_{\text {total }} \cdot l_{e} \cdot \Gamma 2_{k l}^{00}}
\end{aligned}
$$

where $\Gamma_{k l}^{i j}, \Gamma 1_{k l}^{i j}, \Gamma 2_{k l}^{i j}$ in which $i, j=1,2$, determine the order of the derivation of the SF and WF, are the so called connection coefficients [17]. The computation of the connection coefficients is a challenging numerical procedure that is rigorously explained on [31],[32],[7]. Considering the previous equations, the wavelet integrals are given as:

$$
\begin{aligned}
& \int_{0}^{1} \varphi^{(i)}(\xi-k) \varphi^{(j)}(\xi-l) d \xi=\Gamma_{k l}^{i j} \\
& \int_{0}^{1} \varphi^{(i)}(\xi-k) \psi^{(j)}(\xi-l) d \xi=\Gamma 1_{k l}^{i j} \\
& \int_{0}^{1} \psi^{(i)}(\xi-k) \psi^{(j)}(\xi-l) d \xi=\Gamma 2_{k l}^{i j}
\end{aligned}
$$

Due to the orthogonality of SF and WF, there are no coupling mass matrices because:

$$
\left[M_{C F}^{e}\right]=\left[M_{F C}^{e}\right]^{T}=\rho_{\text {total }} l_{e} \int_{0}^{1} \varphi(\xi-k) \cdot \psi(\xi-l) d \xi=0
$$

The mass decoupling and the fact that the mass matrices are consistent and diagonal, leads to crucial computational benefits in the transient dynamic structural analysis. The mass decoupling feature is further discussed in section 3.3 for the hierarchical MR procedure using explicit time integration.

Another important feature of the novel MR-FWD method, is that when the traditional prefinement method is utilized, the mesh remains practically unchanged [17]. Due to the meshless character of the proposed method, increasing the order of Daubechies SF/WF only leads to an increase in the additional grid points to the left side of the physical domain (Figure 2) and the rest of the mesh remains intact.

Moreover, the hierarchical MR solution system that is shown in Eq. (18) can be generalized for $S+1$ resolutions. The $S+1$ resolution stiffness matrices, mass matrices, generalized displacement vectors and load vectors are formed as: 


$$
\left[K_{C C}^{s+1}\right]=\left[\begin{array}{cc}
K_{C C}^{s} & K_{C F}^{s} \\
K_{F C}^{s} & K_{F F}^{s}
\end{array}\right],\left[M_{C C}^{s+1}\right]=\left[\begin{array}{cc}
M_{C C}^{s} & 0 \\
0 & M_{F F}^{s}
\end{array}\right],\left[F_{C}^{s+1}\right]=\left[\begin{array}{c}
F_{C}^{s} \\
F_{F}^{s}
\end{array}\right],\left[\hat{u}_{C C}^{s+1}\right]=\left[\begin{array}{l}
\hat{u}_{C}^{s} \\
\hat{u}_{F}^{s}
\end{array}\right]
$$

It should be noted, that the MR procedure that is presented for the FSDT beam case in Eq. (18) is the same for the rod case, and so is the generalized form of the MR matrices and vectors in Eq. (27).

\subsection{Hierarchical MR process using Explicit time integration scheme}

The hierarchical MR process is shown in Eq. (18) for resolution 1. Essentially, the solution at resolution $S+1$ is the superposition of two different solutions (coarse and fine) at resolution $S$, that are obtained in two independent steps. The first step is always the SR solution that provides the coarse component approximation. For generality, the MR procedure will be described for resolution $S+1$. Considering Eq. (27), the following mass uncoupled MR solution system, that involves both coarse and fine approximation is obtained at resolution $S+1$ :

$$
\left[\begin{array}{cc}
M_{C C}^{s} & 0 \\
0 & M_{F F}^{s}
\end{array}\right]\left\{\begin{array}{l}
\ddot{\hat{u}}_{C}^{s, t} \\
\ddot{\hat{u}}_{F}^{s, t}
\end{array}\right\}+\left[\begin{array}{cc}
K_{C C}^{s} & K_{C F}^{s} \\
K_{F C}^{s} & K_{F F}^{s}
\end{array}\right]\left\{\begin{array}{c}
\hat{u}_{C}^{s, t} \\
\hat{u}_{F}^{s, t}
\end{array}\right\}=\left[\begin{array}{c}
F_{C}^{s, t} \\
F_{F}^{s, t}
\end{array}\right]
$$

The component $\hat{u}_{C}^{s}$ is set as:

$$
\hat{u}_{C}^{s}=\Delta \hat{u}^{s}+\hat{u}_{C C}^{s}
$$

where $\hat{u}_{C C}^{s}$ is the already calculated single-resolution coarse approximation, and $\Delta \hat{u}^{s}$ is the residual between $\hat{u}_{C}^{s}$ and $\hat{u}_{C C}^{s}$, called correction component. It is already mentioned that the multi-resolution coarse component $\hat{u}_{C}^{s}$ is not equal to the single-resolution coarse component $\hat{u}_{C C}^{s}$ because of the stiffness coupling terms. Combining Eqs. (28) and (29), leads to:

$$
\left[\begin{array}{cc}
M_{C C}^{s} & 0 \\
0 & M_{F F}^{s}
\end{array}\right]\left\{\begin{array}{c}
\Delta \ddot{\hat{u}}^{s, t} \\
\ddot{\hat{u}}_{F}^{s, t}
\end{array}\right\}+\left[\begin{array}{cc}
K_{C C}^{s} & K_{C F}^{s} \\
K_{F C}^{s} & K_{F F}^{s}
\end{array}\right]\left\{\begin{array}{c}
\Delta \hat{u}^{s, t} \\
\hat{u}_{F}^{s, t}
\end{array}\right\}=\left[\begin{array}{c}
0 \\
F_{F}^{s, t}-K_{F C}^{s} \hat{u}_{C C}^{s, t}
\end{array}\right]
$$

The diagonality of the total MR mass matrix of Eq. (30) results in two uncoupled equations for the prediction of $\Delta \hat{u}^{s}$ and $\hat{u}_{F}^{s}$, using the central differences explicit time integration scheme. Those are calculated as:

$$
\begin{gathered}
\Delta \hat{u}^{s, t+1}=\frac{\left[M_{C C}^{s}\right]\left(a_{2} \Delta \hat{u}^{s, t}-a_{0} \Delta \hat{u}^{s, t-1}\right)-\left[K_{C C}^{s}\right] \Delta \hat{u}^{s, t}-\left[K_{C F}^{s}\right] \hat{u}_{F}^{s, t}}{a_{0}\left[M_{C C}^{s}\right]} \\
\hat{u}_{F}^{s, t+1}=\frac{F_{F}^{s, t}+\left[M_{F F}^{s}\right]\left(a_{2} \hat{u}_{F}^{s, t}-a_{0} \hat{u}_{F}^{s, t-1}\right)-\left[K_{F C}^{s}\right]\left(\hat{u}_{C C}^{s, t}+\Delta \hat{u}^{s, t}\right)-\left[K_{F F}^{s}\right] \hat{u}_{F}^{s, t}}{a_{0}\left[M_{F F}^{s}\right]}
\end{gathered}
$$

where $a_{0}=1 / \Delta t^{2}$ and $a_{2}=2 / \Delta t^{2}$, in which $\Delta \mathrm{t}$ is the time step for the explicit dynamic solver. The superposition of the three components $\hat{u}_{C C}^{s}, \Delta \hat{u}^{s}$ and $\hat{u}_{F}^{s}$ at resolution $S$, forms the coarse approximation of the next resolution $S+1$, as:

$$
\hat{u}_{C C}^{s+1, t+1}=\hat{u}_{C C}^{s, t+1}+\Delta \hat{u}^{s, t+1}+\hat{u}_{F}^{s, t+1}
$$




\section{NUMERICAL RESULTS}

The efficiency and the functionalities of the MR-FWD method are evaluated towards the simulation of longitudinal and guided waves in homogeneous and heterogeneous aluminum rods and laminated carbon/epoxy beams. The mechanical properties of the involved materials are shown in Table 1.

\begin{tabular}{lccccc}
\hline & $\mathrm{E}_{11}(\mathrm{GPa})$ & $\mathrm{E}_{22}=\mathrm{E}_{33}(\mathrm{GPa})$ & $\mathrm{G}_{12}=\mathrm{G}_{23}=\mathrm{G}_{13}(\mathrm{GPa})$ & $\mathrm{v}_{12}=\mathrm{v}_{13}=\mathrm{v}_{23}$ & $\rho\left(\mathrm{kg} / \mathrm{m}^{3}\right)$ \\
\hline Aluminum & 70 & 70 & 26.923 & 0.3 & 2700 \\
Damaged Aluminum & 7 & 7 & 2.6923 & 0.3 & 2700 \\
Carbon/Epoxy & 120 & 7.9 & 5.5 & 0.3 & 1580 \\
Damaged Carbon/Epoxy & 114 & 7.505 & 5.225 & 0.3 & 1580 \\
\hline
\end{tabular}

Table 1: Material properties.

\subsection{Homogeneous Aluminum Rod}

An aluminum rod with length $l=4 \mathrm{~m}$, cross section $A=10^{-4} \mathrm{~m}^{2}$ and mechanical properties that are shown in Table 1, is modeled. The rod is clamped at its left edge and it is excited at its center $(x=2 m)$, by a 5 -cycle tone burst with $25 \mathrm{kHz}$ central frequency. The analysis duration is 0.3 milliseconds $(\mathrm{ms})$. The effectiveness of the MR hierarchical procedure is investigated for resolution up to 2, for DB3 wavelet-based elements. A converged 3-node FE analysis with 401 nodes is considered the reference solution. It is worth mentioning that both the 3-node FE and the DB3 wavelet-based elements, are quadratic approximation elements. The hierarchical process is shown in Figure 3, following top-down sequence.

The first step of the analysis is the SR approximation $\left(C^{0}\right)$ that involves 54 grid points. It is obvious that this coarse solution $\hat{u}_{C C}^{0}$ diverges a lot from the reference solution. Moving to resolution 1 , the components $\Delta \hat{u}^{0}$ and $\hat{u}_{F}^{0}$ are calculated and superimposed to $\hat{u}_{C C}^{0}$, using the same grid points. In that way, the coarse solution at resolution $1\left(C^{l}\right)$ is obtained, and it is shown that crucial correction is provided by $\Delta \hat{u}^{0}$ and $\hat{u}_{F}^{0}$. The same procedure is repeated for resolution 2, but the shrinking of the DB3 WF requires the use of 104 grid points, for the calculation of $\Delta \hat{u}^{1}$ and $\hat{u}_{F}^{1}$. The total summation of the above 5 components $\left(\hat{u}_{C C}^{0}, \Delta \hat{u}^{0}, \hat{u}_{F}^{0}, \Delta \hat{u}^{1}, \hat{u}_{F}^{1}\right)$ provides the overall solution at resolution $2\left(C^{2}\right)$, which has converged to the reference solution. It can be concluded, that the components $\Delta \hat{u}^{s}$ mainly improve the frequency and the time of flight of the wave packets, whereas the components $\hat{u}_{F}^{s}$ provide a small correction on the amplitude of the wave packets. The functionalities of the fine components will be extensively assessed at the inhomogeneous cases. 


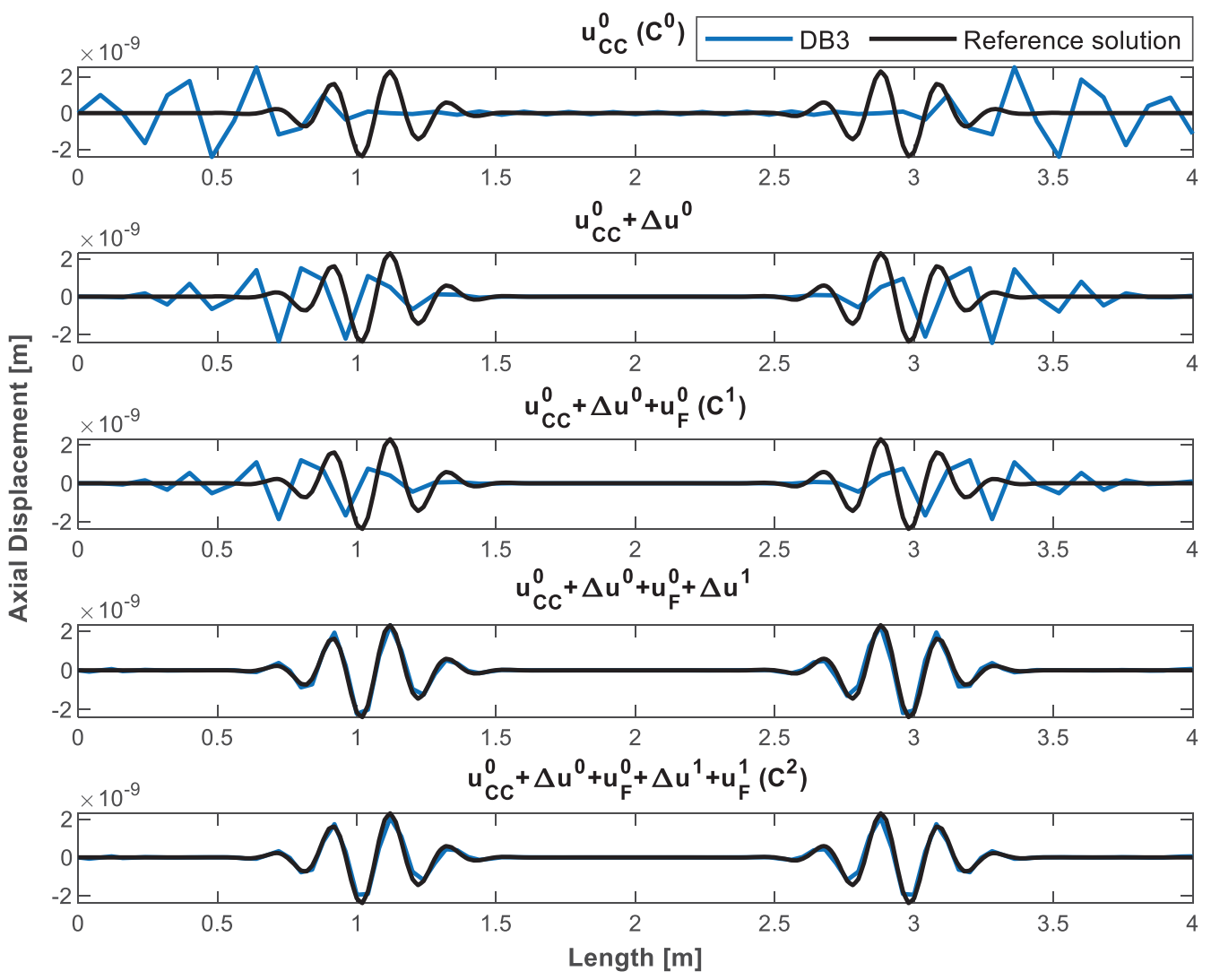

Figure 3: Predicted axial displacement field of the aluminum rod. The MR hierarchical procedure is shown from resolution 0 to resolution 2 (top-down sequence).

The MR hierarchical process demonstrates substantial reduction in the computational effort, because not only the required grid points for a converged solution are much fewer compared to the 3-node FE, but also the mass diagonality permits the initial $2 N \times 2 N$ algebraic system (Eq. (28)) to be solved as two $N \times N$ algebraic systems (Eq. (31) and (32)), using explicit time integration. Considering the computational cost in terms of floating-point operations (flops), a matrix inversion using LU decomposition requires approximately $2 n^{3} / 3$ flops. So, inverting two $N \times N$ matrices requires approximately 75\% less flops in comparison with inverting a $2 N \times 2 N$ matrix. In that way, additional computational benefits arise from the hierarchical MR procedure, apart from the exploitation of the already calculated coarse solution.

\subsection{Laminated Composite Beam}

A $1 \mathrm{~m}$ long unidirectional [0]8 carbon/epoxy beam with cross section $A=10^{-4} \mathrm{~m}^{2}$ and mechanical properties shown in Table 1, is modeled. The beam is clamped at its left edge and it is transversely excited at its center $(x=0.5 \mathrm{~m})$, by a 5 -cycle tone burst with $50 \mathrm{kHz}$ central frequency. The analysis duration is $0.15 \mathrm{~ms}$. The simulation of antisymmetric guided waves is more challenging than the simulation of pressure waves, owing to the smaller wavelengths and the dispersive nature of the antisymmetric mode. In this case, the effectiveness of the pmethod for resolution 1 solutions $\left(C^{l}\right)$ is demonstrated. As shown in Figure 4, MR-FWD models with DB3, DB6, DB8 and DB11 wavelet-based elements are used to predict the transverse displacement field of the composite beam. The reference solution is a converged and validated FWD analysis $\left(C^{0}\right)$ that employs DB3 elements with 434 grid points. 


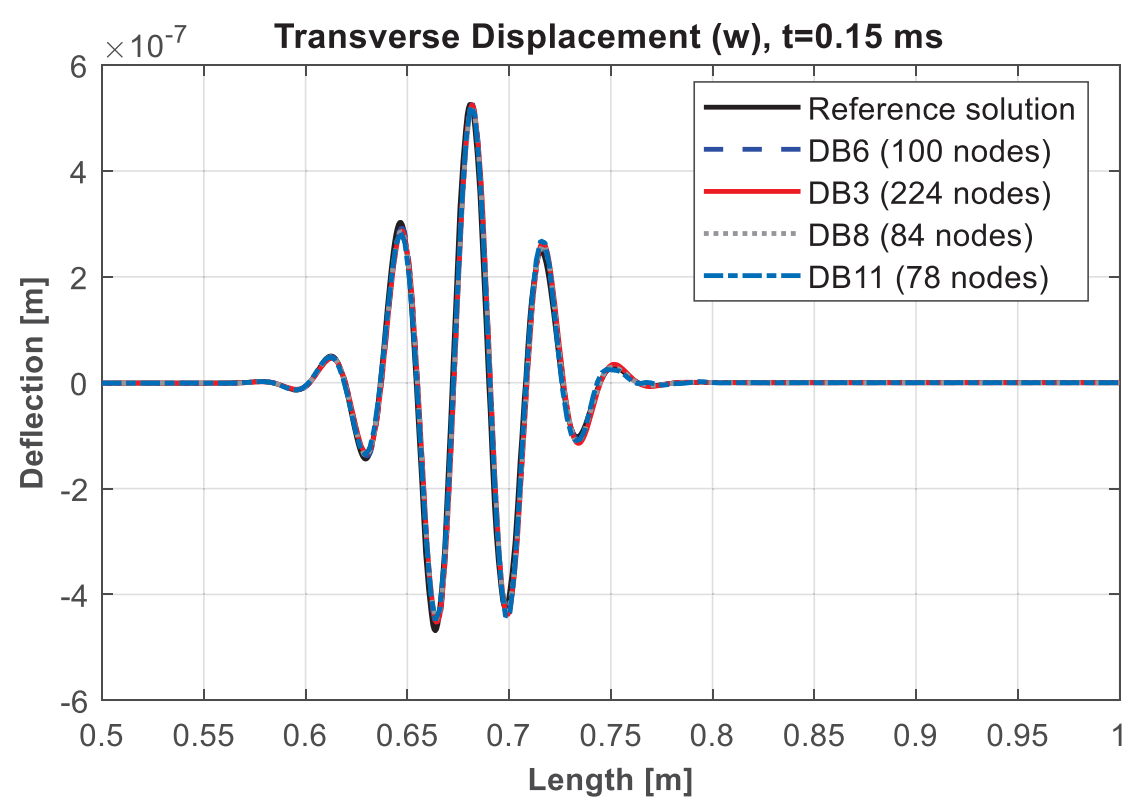

Figure 4: Predicted transverse displacement of the right half of the laminated beam. Minimum number of nodes required for a converged solution are illustrated for each discretization case inside the parentheses.

The superiority of the high order MR DB elements is depicted in Figure 4, where it is obvious that higher order MR models require substantially fewer nodes to obtain an accurate solution. Moreover, observing the case of DB3 discretization, the MR hierarchical method demonstrates remarkable precision and critically decreases the already decreased computational effort required by the single-resolution FWD method. Considering the grid points needed for a converged solution, there is a reduction of almost $50 \%$ for the $C^{l}$ DB3 case and up to $82 \%$ for the $C^{l}$ DB11 case, compared to the $C^{0}$ DB3 analysis. Finally, it should be noted that the wavelet-based FSDT beam elements do not exhibit shear locking effects, which is another significant advantage compared to traditional shear beam FE [17].

\subsection{Inhomogeneous Aluminum Rod}

An inhomogeneous aluminum rod with length $l=4 \mathrm{~m}$ and cross section $A=10^{-4} \mathrm{~m}^{2}$ is modeled. The rod consists of two different materials, aluminum, and damaged aluminum, and their mechanical properties are shown in Table 1. The damaged area spans from $2 m \leq x \leq 2.2 m$, as depicted with dark grey in Figure 5a. The rod is clamped at the left edge and it is excited at its right free end $(x=4 m)$, by a 5 -cycle tone burst with $25 \mathrm{kHz}$ central frequency. The duration of the simulation is $0.8 \mathrm{~ms}$. The reference solution is a converged SR analysis of 800 DB3 elements. Figure 5b depicts the axial displacement of the MR process, for $200 \mathrm{DB} 3$ elements and resolutions from 0 to 2.

It is obvious that the initial scale of analysis $\left(C^{0}\right)$ exhibits substantial error compared to the reference solution. Moving up to the $C^{l}$ there is crucial correction of the predicted displacement field, but there is still divergence from the reference solution. Finally, the $C^{2}$ solution shows excellent agreement with the reference solution. The examination of the fine components introduces certain critical results and reveals some of their extraordinary benefits. 
(a)

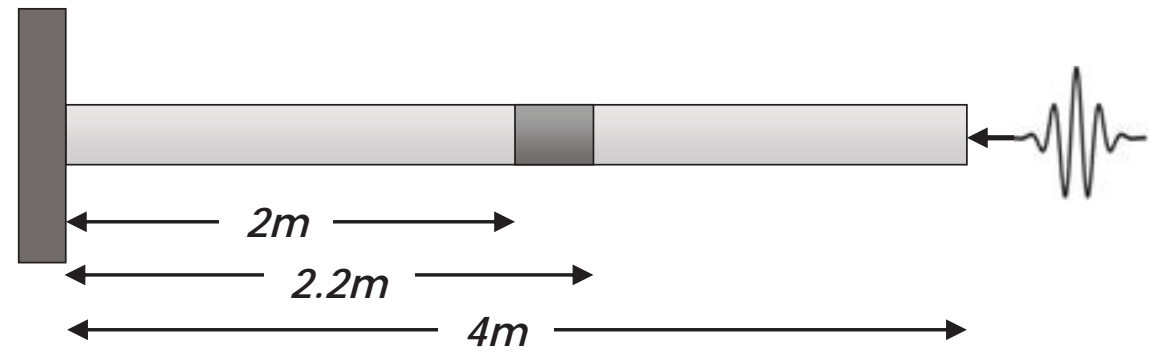

(b)

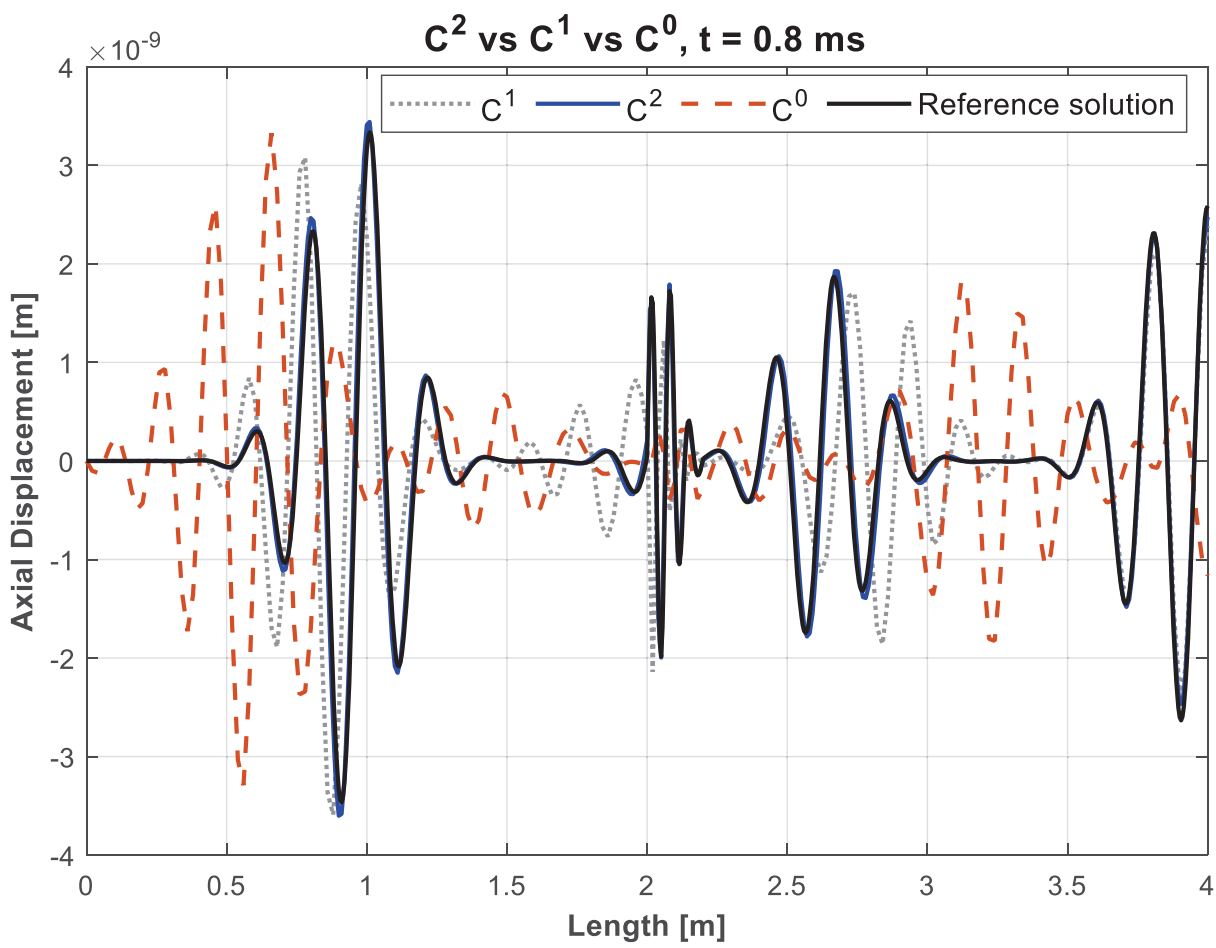

Figure 5: (a) Wave propagation in damaged rod structure. (b) Axial displacement field of the inhomogeneous rod for resolutions 0,1 and 2 .
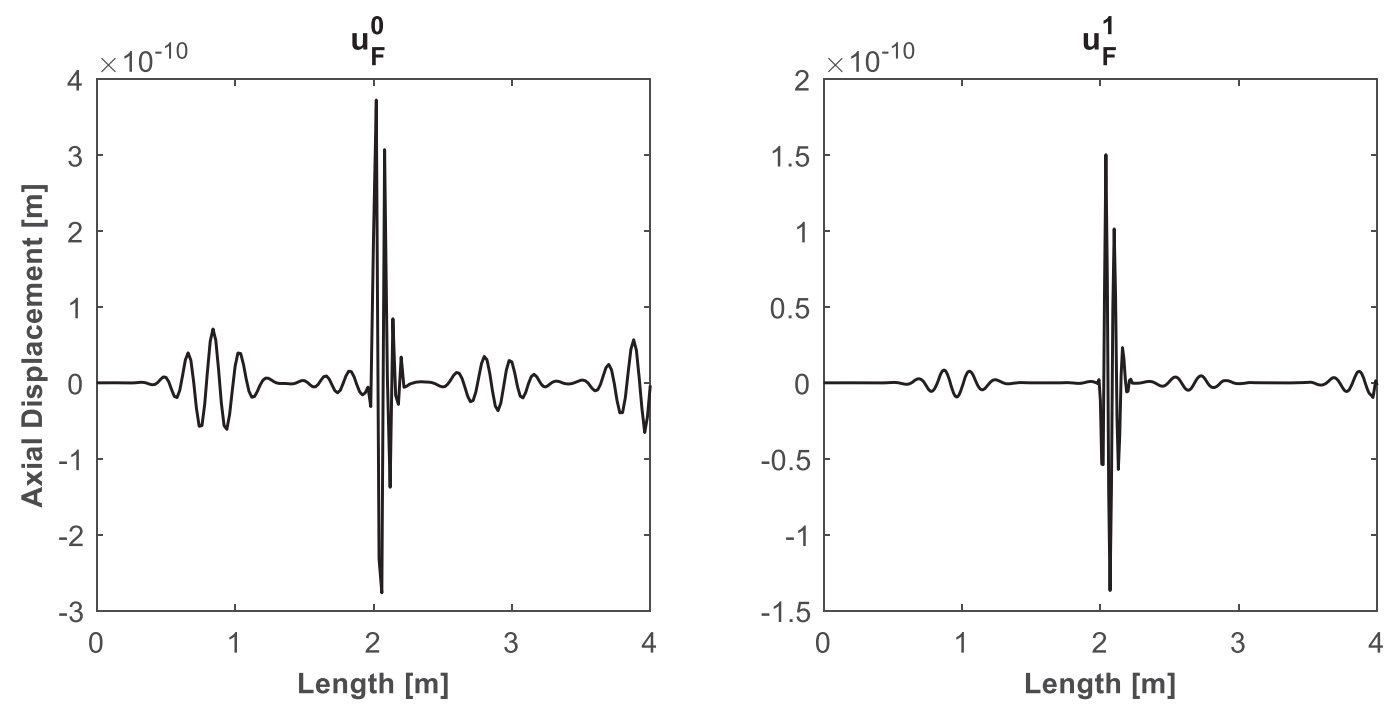

Figure 6: Contribution of the fine components of resolutions 0 (left) and 1 (right), in which the inhomogeneity is precisely detected and also the higher wavenumber inside the damaged area is accurately predicted. 
As shown in Figure 6, the fine components $\hat{u}_{F}^{0}, \hat{u}_{F}^{1}$ exactly localize the span of the damage and capture the higher wavenumbers inside the inhomogeneity due to the high frequency nature of Daubechies WF. In particular, the $\hat{u}_{F}^{0}$ component uses the same nodes as the $\hat{u}_{C C}^{0}$, but efficiently approaches the smaller wavelengths that arise from the lower Young modulus of the damaged region. Apparently, the $\hat{u}_{F}^{1}$ component that uses double nodes in comparison with the $\hat{u}_{C C}^{0}$ solution, precisely predicts the higher wavenumbers inside the inhomogeneity. Thus, besides the critical benefits of the MR-FWD method in terms of accuracy and computational effectiveness, there are additional gains concerning the fine components, which are extremely sensitive in detecting discontinuities and can numerically simulate steep gradients, even at coarse discretization cases.

\subsection{Damaged Composite Beam}

A $1 m$ long cross-ply [0/90/0/90]s carbon/epoxy beam with cross section $A=10^{-4} \mathrm{~m}^{2}$ is modeled. The beam consists of two different regions, the pristine carbon/epoxy, and the damaged carbon/epoxy region. The mechanical properties of those materials are shown in Table 1 . The damage is assumed to induce $5 \%$ degradation to all the elastic moduli. The damaged area ranges from $0.75 m \leq x \leq 0.8 m$, as illustrated with dark grey in Figure $7 \mathrm{a}$. The beam is clamped at its left edge and it is excited at its center $(x=0.5 \mathrm{~m})$, by a 5 -cycle tone burst with $50 \mathrm{kHz}$ central frequency. The analysis duration is $0.2 \mathrm{~ms}$. The efficiency of the MR hierarchical scheme is highlighted in the previous sections. In this section, the sensitivity of the fine components is exhibited. A converged discretization of 314 grid points of DB8 elements is used for resolution up to 1 .

Figure $7 \mathrm{~b}$ depicts the predicted deflection of the beam for the converged solutions $C^{0}, C^{1}$ and the fine component $w_{F}^{0}$. The objective of this analysis is not to emphasize the computational gains of the MR-FWD method in terms of the required grid points, since $C^{0}$ and $C^{l}$ approximations are in excellent agreement. Hence, moving up to resolution 1 does not offer any correction at the $C^{0}$ solution. Nevertheless, the existence of the damaged area is not perceptible at $C^{0}$ or $C^{l}$ solution, even though the right wave packet passes through the heterogeneity at $t=2 \mathrm{~ms}$. The fine component $w_{F}^{0}$ provides precise detection of the interfaces between the damaged and the pristine composite beam despite the small degradation of its mechanical properties by $5 \%$. In this case, the fine component does not capture a dissimilar wavenumber inside the heterogeneity, because of the insignificant difference among the material properties of the damaged and the pristine carbon/epoxy. Also, $w_{F}^{0}$ does not practically participate in the total solution in quantitative terms, since the $C^{0}$ solution is meticulous due to the dense discretization. However, the fine solution can exactly localize the boundaries between the two materials, introducing a pioneering additional property of the proposed numerical method. 
(a)

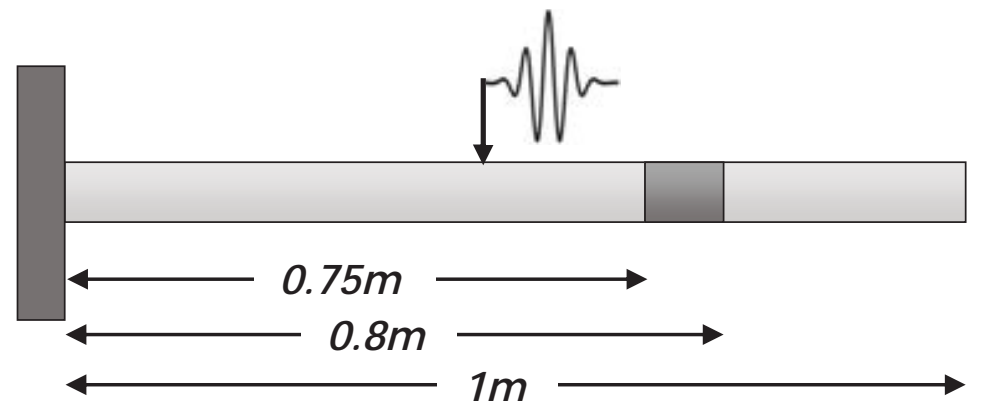

(b)
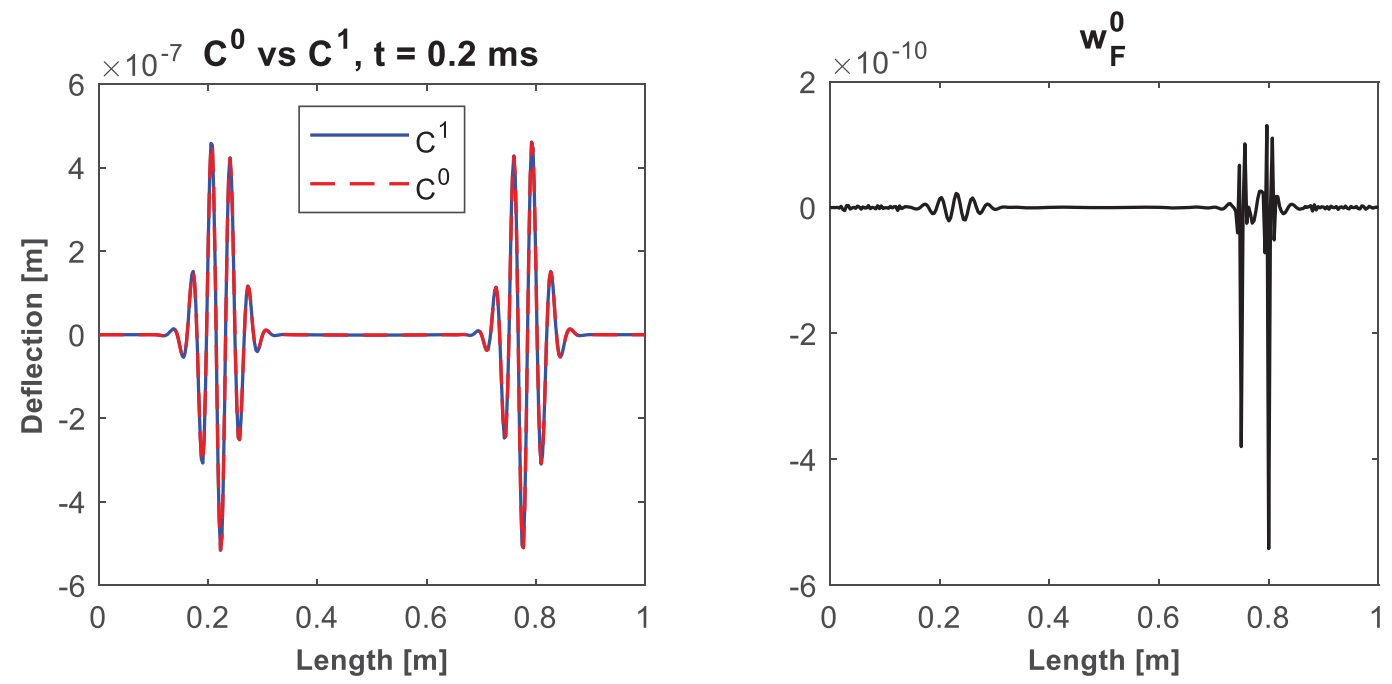

Figure 7: (a) Wave propagation in damaged composite beam. (b) $C^{0}$ and $C^{l}$ solutions are converged (left) but the inhomogeneity is not visible. The fine component accurately delimits the damage span (right).

\section{CONCLUSIONS}

A novel Multi-resolution hierarchical wavelet-based method for the efficient simulation of transient dynamic response of rods and beams, is presented. Taking advantage of the remarkable mathematical properties and the unique MR capability of Daubechies wavelet and scaling functions, the proposed 1D MR-FWD method has been developed, as an expansion of the single-resolution FWD method. The MR approximation includes two types of variables, the coarse and fine wavelet coefficients of each resolution. The implementation of the MR approximation at the equations of motion, yields a MR solution system that involves both coarse and fine resolution degrees of freedom. The hierarchical process starts with the coarse solution $\left(C^{0}\right)$, which basically is the FWD method, and then a fine approximation $\left(F^{0}\right)$ is calculated and superimposed on the coarse solution, forming the $1^{\text {st }}$ improved solution $\left(C^{l}\right)$. This procedure can be continued until the desired level of accuracy is achieved. Therefore, the proposed MR-FWD method exploits the non-converged solutions that are obtained at coarser resolutions, compared to traditional numerical methods that discard them. Due to the orthogonality of the Daubechies wavelet and scaling functions, the consistent mass matrices of each resolution are diagonal or at least block diagonal. Also, the mass diagonality results in decoupling of the discretized dynamic equations with the use of explicit time integration scheme. It should be emphasized, that the approximation $C^{l}$ provides a much more accurate prediction than $C^{0}$, using the same grid points as the approximation $C^{0}$. Additionally, it should be noted, that performing p-refinement to the proposed method, leaves the mesh practically unaffected. The precision and computational efficiency of the developed method were evaluated by com- 
paring the predicted longitudinal and guided wave responses of homogeneous and heterogeneous rods and beams, with validated FE and single-resolution FWD models.

The numerical results demonstrated 4 key advantages of the MR-FWD method: (1) the MR property of Daubechies wavelets leads to a hierarchical MR set of dynamic equations, that introduce a new type of refinement which utilizes the solution of an already obtained coarser analysis; (2) the hierarchical MR procedure in synergy with the advantageous use of the classical p-method, enables the exact solution of demanding transient dynamic problems, using smaller size algebraic systems compared to the single-resolution FWD method; (3) the consistent diagonal mass matrices of each resolution in conjunction with the mass decoupling, lead to faster analyses with the use of explicit time integration; and (4) the fine components of the MR analysis introduce additional benefits to the MR-FWD method, such as the efficient capture of the higher wavenumbers and the exact localization of inhomogeneities and interfaces. Future work may focus on the inclusion of a higher order layerwise theory to the proposed MR-FWD method, for the accurate simulation of both symmetric and antisymmetric modes in thick composites and sandwich structures.

\section{REFERENCES}

[1] S. Jaffard, "Wavelet methods for fast resolution of elliptic problems," SIAM J. Numer. Anal., vol. 29, no. 4, pp. 965-986, 1992, doi: 10.1137/0729059.

[2] R. D. Patton and P. C. Marks, "One-dimensional finite elements based on the Daubechies family of wavelets," AIAA J., vol. 34, no. 8, pp. 1696-1698, 1996, doi: 10.2514/3.13291.

[3] J. Ma, J. Xue, S. Yang, and Z. He, "A study of the construction and application of a Daubechies wavelet-based beam element," Finite Elem. Anal. Des., vol. 39, no. 10, pp. 965-975, 2003, doi: 10.1016/S0168-874X(02)00141-5.

[4] L. A. Díaz, M. T. Martín, and V. Vampa, "Daubechies wavelet beam and plate finite elements," Finite Elem. Anal. Des., vol. 45, no. 3, pp. 200-209, 2009, doi: 10.1016/j.finel.2008.09.006.

[5] R. B. Burgos, M. A. Cetale Santos, and R. R. E. Silva, "Deslauriers-Dubuc interpolating wavelet beam finite element," Finite Elem. Anal. Des., vol. 75, pp. 71-77, 2013, doi: 10.1016/j.finel.2013.07.004.

[6] R. B. Burgos and M. A. C. Santos, "Finite Elements Based on Deslauriers-Dubuc Wavelets for Wave Propagation Problems," Appl. Math., vol. 07, no. 14, pp. 14901497, 2016, doi: 10.4236/am.2016.714128.

[7] J. Ko, A. J. Kurdila, and M. S. Pilant, "A class of finite element methods based on orthonormal, compactly supported wavelets," Comput. Mech., vol. 16, no. 4, pp. 235244, 1995, doi: 10.1007/BF00369868.

[8] M. Mitra and S. Gopalakrishnan, "Spectrally formulated wavelet finite element for wave propagation and impact force identification in connected 1-D waveguides," Int. J. Solids Struct., vol. 42, no. 16-17, pp. 4695-4721, 2005, doi: 10.1016/j.ijsolstr.2005.02.007.

[9] D. Samaratunga, R. Jha, and S. Gopalakrishnan, "Wavelet spectral finite element for wave propagation in shear deformable laminated composite plates," Compos. Struct., 
vol. 108, no. 1, pp. 341-353, 2014, doi: 10.1016/j.compstruct.2013.09.027.

[10] L. Pahlavan, C. Kassapoglou, and Z. Gürdal, "Spectral formulation of finite element methods using Daubechies compactly-supported wavelets for elastic wave propagation simulation," Wave Motion, vol. 50, no. 3, pp. 558-578, 2013, doi: 10.1016/j.wavemoti.2012.12.006.

[11] J. W. Xiang, X. F. Chen, Z. J. He, and H. B. Dong, "The construction of 1D wavelet finite elements for structural analysis," Comput. Mech., vol. 40, no. 2, pp. 325-339, 2007, doi: 10.1007/s00466-006-0102-5.

[12] Z. B. Yang, X. F. Chen, Y. Xie, H. Zuo, H. H. Miao, and X. W. Zhang, "Wave motion analysis and modeling of membrane structures using the wavelet finite element method," Appl. Math. Model., vol. 40, no. 3, pp. 2407-2420, 2016, doi: 10.1016/j.apm.2015.09.071.

[13] X. Zhang, X. Chen, X. Wang, and Z. He, "Multivariable finite elements based on Bspline wavelet on the interval for thin plate static and vibration analysis," Finite Elem. Anal. Des., vol. 46, no. 5, pp. 416-427, 2010, doi: 10.1016/j.finel.2010.01.002.

[14] X. Wei, W. Chen, and B. Chen, "B-spline wavelet on interval finite element method for static and vibration analysis of stiffened flexible thin plate," Comput. Mater. Contin., vol. 52, no. 1, pp. 53-71, 2016, doi: 10.3970/cmc.2016.052.053.pdf.

[15] W. Shen, D. Li, and J. Ou, "Modeling dispersive waves in cracked rods using the wavelet-based higher-order rod elements," Int. J. Mech. Sci., vol. 166, no. October 2019, 2020, doi: 10.1016/j.ijmecsci.2019.105236.

[16] X. Xue, X. Chen, X. Zhang, and B. Qiao, "Hermitian plane wavelet finite element method: Wave propagation and load identification," Comput. Math. with Appl., vol. 72, no. 12 , pp. 2920-2942, 2016, doi: 10.1016/j.camwa.2016.10.019.

[17] C. V. Nastos, T. C. Theodosiou, C. S. Rekatsinas, and D. A. Saravanos, "A finite wavelet domain method for the rapid analysis of transient dynamic response in rods and beams," C. - Comput. Model. Eng. Sci., vol. 107, no. 5, pp. 379-409, 2015, doi: 10.3970/cmes.2015.107.379.

[18] C. V. Nastos, T. C. Theodosiou, C. S. Rekatsinas, and D. A. Saravanos, "A 2D Daubechies finite wavelet domain method for transient wave response analysis in shear deformable laminated composite plates," Comput. Mech., vol. 62, no. 5, pp. 1187-1198, 2018, doi: 10.1007/s00466-018-1558-9.

[19] C. V. Nastos and D. A. Saravanos, "A finite wavelet domain method for wave propagation analysis in thick laminated composite and sandwich plates," Wave Motion, vol. 95, p. 102543, 2020, doi: 10.1016/j.wavemoti.2020.102543.

[20] S. G. Mallat, "A theory for multiresolution signal decomposition: The wavelet representation," Fundam. Pap. Wavelet Theory, vol. II, no. 7, pp. 494-513, 2009, doi: $10.1515 / 9781400827268.494$.

[21] I. Daubechies, “Ten Lectures on Wavelets - Ingrid Daubechies (1992).pdf.” 1998.

[22] S. M. Quraishi and K. Sandeep, "Multiscale modeling of beam and plates using customized second-generation wavelets," J. Eng. Math., vol. 83, no. 1, pp. 185-202, 2013, doi: 10.1007/s10665-012-9579-4.

[23] G. W. Jang, J. E. Kim, and Y. Y. Kim, "Multiscale Galerkin method using 
interpolation wavelets for two-dimensional elliptic problems in general domains," Int. J. Numer. Methods Eng., vol. 59, no. 2, pp. 225-253, 2004, doi: 10.1002/nme.872.

[24] X. Liu, G. R. Liu, J. Wang, and Y. Zhou, "A wavelet multiresolution interpolation Galerkin method for targeted local solution enrichment," Comput. Mech., vol. 64, no. 4, pp. 989-1016, 2019, doi: 10.1007/s00466-019-01691-6.

[25] S. Tanaka, H. Suzuki, S. Ueda, and S. Sannomaru, "An extended wavelet Galerkin method with a high-order B-spline for 2D crack problems," Acta Mech., vol. 226, no. 7, pp. 2159-2175, 2015, doi: 10.1007/s00707-015-1306-6.

[26] X. Liu, G. R. Liu, J. Wang, and Y. Zhou, "A wavelet multiresolution interpolation Galerkin method with effective treatments for discontinuity for crack growth analyses," Eng. Fract. Mech., vol. 225, no. November 2019, p. 106836, 2020, doi: 10.1016/j.engfracmech.2019.106836.

[27] S. Tanaka, S. Sannomaru, M. Imachi, S. Hagihara, S. Okazawa, and H. Okada, "Analysis of dynamic stress concentration problems employing spline-based wavelet Galerkin method," Eng. Anal. Bound. Elem., vol. 58, pp. 129-139, 2015, doi: 10.1016/j.enganabound.2015.04.003.

[28] W. Shen, D. Li, and J. Ou, "Dispersion Analysis of Multiscale Wavelet Finite Element for 2D Elastic Wave Propagation," J. Eng. Mech., vol. 146, no. 4, pp. 1-17, 2020, doi: 10.1061/(ASCE)EM.1943-7889.0001756.

[29] B. Li and X. Chen, "Wavelet-based numerical analysis: A review and classification," Finite Elem. Anal. Des., vol. 81, pp. 14-31, 2014, doi: 10.1016/j.finel.2013.11.001.

[30] W. Lin, N. Kovvali, and L. Carin, "Direct algorithm for computation of derivatives of the Daubechies basis functions," Appl. Math. Comput., vol. 170, no. 2, pp. 1006-1013, 2005, doi: 10.1016/j.amc.2004.12.038.

[31] M.-Q. CHEN, C. HWANG, and Y.-P. SHIH, "the Computation of Wavelet-Galerkin Approximation on a Bounded Interval," Int. J. Numer. Methods Eng., vol. 39, no. 17, pp. 2921-2944, 1996, doi: 10.1002/(sici)1097-0207(19960915)39:17<2921::aidnme983>3.3.co;2-4.

[32] T. Zhang, Y. C. Tian, M. O. Tadé, and J. Utomo, "Comments on 'The computation of wavelet-Galerkin approximation on a bounded interval,", Int. J. Numer. Methods Eng., vol. 72, no. 2, pp. 244-251, 2007, doi: 10.1002/nme.2022. 